\title{
Professional/Amateur Cooperation in the Study of Variable Stars as Illustrated by the Fourth Edition of the GCVS
}

\author{
M.S. Frolov \\ Astronomical Council, USSR Academy of Sciences, \\ 48 Pyatnitskaya ul., 109017, Moscow, U.S.S.R.
}

Let us divide variable stars into two main groups: the first "classical" group, includes objects known for a long time, such as Cepheids, RR-Lyrae stars, Miras, cataclysmic variables, eclipsing binaries, etc. The second group includes micropulsating variables of $\delta$ Scuti and $\beta$ Cephei types, magnetic variables, rotating variables of BY Draconis type, etc.

Historically, the contribution of amateurs in investigating the first group was very significant, and it continues to increase. On the other hand, involvement in studying the second group of stars was practically equal to zero some years ago, but today one can see the beginnings of an expansion of amateur work on this second group of variables - among brighter objects, of course. One reason is the beginning of cooperation between amateurs and professional astronomers having powerful instruments.

Another good example of the fruitful collaboration between amateur and professional astronomers over many years is in the preparation of the General Catalogue of Variable Stars (the GCVS). This Catalogue is well-known around the world, not only to variable-star investigators, but now also to a considerable number of modern astrophysicists.

Since the publication of the first edition of the Catalogue in 1948, two other editions have been prepared and fourteen Supplements, three editions of the Catalogue of Suspected Variables, twenty-five Name-Lists of New Variable Stars and three volumes of the fourth edition of the GCVS. The fourth edition, including the NSV-catalogue, will contain information of fifty thousand variable stars in our Galaxy and in external galaxies.

The team of authors of the GCVS makes wide use of the data on variable stars obtained by amateurs. Among the principal bibliographic references in the GCVS we can find such amateur publications as the Journal of the AAVSO, AAVSO Abstracts, Bulletin of the AFOEV, publications of GEOS, RASNZ, BAA VSS, publications of Italian amateur astronomers, and BBSAG (Switzerland). We continue to urgently need maxima of Miras, traditionally published by the AAVSO, minima of eclipsing binaries (BBSAG), maxima of RR-Lyrae stars from different amateur sources, results of regular studies of cataclysmic variables from AAVSO and RASNZ bulletins. For example, the contribution of amateurs in the field of Miras amounts to about $90 \%$. 
Today there are few amateur observations that we can use for variables in the second group, because microvariables can be successfully investigated only with high-precision photoelectric data. Only a wide distribution of photoelectric photometers and CCD-photometers among astronomers can seriously change this situation. In this respect we see very good prospects from the cooperation between professionals and amateurs. A good example of such cooperation is the IAPPP.

The team of authors of the GCVS is very indebted to all the amateur astronomers for the useful data that help us to prepare our Catalogue. 